\title{
Rede de cuidado a crianças e adolescentes em sofrimento psíquico: ações de promoção à saúde*
}

\section{Care network for children and adolescents in psychic suffering: health promotion actions}

\author{
Mayara Francelle Oliveira Barata ${ }^{1}$, Keise Bastos Gomes da Nóbrega ${ }^{2}$, \\ Kátia Cilene Silva de Jesus ${ }^{3}$, Maria Luiza Lopes Timóteo de Lima ${ }^{4}$, \\ Vera Lucia Dutra Facundes ${ }^{5}$
}

http://dx.doi.org/10.11606/issn.2238-6149.v26i2p225-233

\begin{abstract}
Barata MFO, Nóbrega KBG, Jesus KCS, Lima MLLT, Facundes VLD. Rede de cuidado a crianças e adolescentes em sofrimento psíquico: ações de promoção à saúde. Rev Ter Ocup Univ São Paulo. 2015 maio-ago.;26(2):225-33.
\end{abstract}

RESUMO: Este artigo se propõe a investigar a rede de cuidado de crianças e adolescentes em sofrimento psíquico e a descrever ações que visam à promoção da saúde mental, a partir de uma intervenção territorial. Trata-se de um estudo qualitativo, baseado no desenho da pesquisa-ação, cuja amostra foi constituída por seis crianças e nove adolescentes residentes no território adscrito de quatro Unidades de Saúde da Família. Utilizou-se uma entrevista semiestruturada contendo dados socioeconômicos, demográficos, e as relações na rede familiar e de cuidados. Também foi utilizado o diário de campo para transcrição das observações de intervenções realizadas no domicílio e na comunidade, na perspectiva da atenção primária à saúde. Foram identificadas falhas na efetivação do brincar, no desempenho do lazer, dificuldades escolares e alterações comportamentais; tais aspectos têm sido prejudicados por relações familiares conflituosas e precárias condições de vida. Verificou-se que a escola e os vizinhos são elementos de apoio na rede de cuidados das crianças e adolescentes, sendo focos das intervenções territoriais. $\mathrm{O}$ cuidado em saúde mental requer uma rede integrada, com ferramentas efetivas para a promoção da autonomia e participação social destes sujeitos.

DESCRITORES: Atenção primária à saúde; Assistência à saúde; Cuidado da criança; Saúde do adolescente; Saúde mental; Terapia ocupacional.
Barata MFO, Nóbrega KBG, Jesus KCS, Lima MLLT, Facundes VLD. Care network for children and adolescents in psychic suffering: health promotion actions. Rev Ter Ocup Univ São Paulo. 2015 May-Aug.;26(2):225-33.

ABSTRACT: The purpose of this article is to investigate the care network for children and adolescents in psychological suffering and to describe actions aimed at promoting mental health from a territorial intervention. This is a qualitative study based on an action research design whose sample was composed of 6 children and 9 adolescents living in the ascribed territory of four Family Health Units. A semistructured interview was used, containing socioeconomic and demographic data and the relations in family and care network. The field log was also used for the transcription of the observations of interventions performed at home and in the community, from the perspective of primary health care. Flaws in the achievement of the play and the leisure performance were identified, as well as learning difficulties and behavioral changes; these aspects have been hindered by conflicting family relationships and poor living conditions. It was found that the school and the neighbors, which are the focus of the territorial interventions, are supporting elements in the care network of children and adolescents. Mental health care requires an integrated network with effective tools to promote autonomy and social participation of these subjects.

KEYWORDS: Primary health care; Delivery of health care; Child care; Adolescent health; Mental health; Occupational therapy.

\footnotetext{
* Artigo referente à tese de conclusão de curso de Mayara Francelle Oliveira Barata, do curso de Terapia Ocupacional da Universidade Federal de Pernambuco. A pesquisa fez parte do programa PIBIC/CNPQ, sobre orientação das docentes Keise Nóbrega e Vera Facundes.

1. Terapeuta ocupacional, Hospital Universitário Oswaldo Cruz.

2. Docente do curso de Terapia Ocupacional da Universidade Federal de Pernambuco - UFPE.

3. Terapeuta ocupacional da Prefeitura da Cidade do Recife, PE.

4. Docente do curso de Fonoaudiologia da Universidade Federal de Pernambuco - UFPE.

5. Docente do curso de Terapia Ocupacional da Universidade Federal de Pernambuco - UFPE.

Endereço para correspondência: Av. Ministro Marcos Freire, 4411. Recife, PE.
} 


\section{INTRODUÇÃO}

$\mathrm{A}$ atenção integral à saúde mental infantojuvenil é constituída a partir de uma rede ampliada de cuidados, que deve articular serviços de diferentes setores, capazes de desenvolver a assistência integral. A atenção básica, a educação e a assistência social se constituem setores estratégicos na política de saúde mental para crianças e adolescentes, pois contribuem para a promoção da saúde e prevenção de agravos, no processo de identificação e monitoramento de casos, e viabilizam o acesso da população à rede de cuidados ${ }^{1}$.

No Brasil, estima-se que $10 \%$ a $20 \%$ da população infantojuvenil sofram com transtornos mentais e, desses, $3 \%$ a $4 \%$ precisem de tratamento intensivo. Estudos realizados entre 1980 e 2006 registraram prevalência de $12,6 \%$ a $35,2 \%$ quando os informantes foram os pais ou a criança. Ao ser utilizada uma entrevista diagnóstica, a taxa variou entre $7 \%$ e $12,7 \%{ }^{1-2}$.

Crianças e adolescentes com transtornos mentais podem ter sérios prejuízos em seu desempenho funcional, que corresponde à capacidade do indivíduo em realizar atividades de seu cotidiano de maneira satisfatória e apropriada para cada etapa de desenvolvimento. $\mathrm{Na}$ população infantil, a dificuldade em realizar essas atividades geralmente é demonstrada pelas falhas na estrutura social, afetando principalmente os papéis de brincar e de estudante ${ }^{3-4}$.

Percebe-se, portanto, que o sofrimento psíquico traz implicações que não se restringem à patologia da criança ou do adolescente. Por isso, há a necessidade da abordagem integral que envolva o usuário, sua família, a comunidade e os serviços de apoio. Trata-se de enfrentar os graves comprometimentos psíquicos com compromissos coletivos, em redes sociais de proteção. A vulnerabilidade e o risco social de crianças e de adolescentes com transtornos mentais diminuem quanto mais se ampliam os compromissos coletivos. Portanto, precisam ter em torno de si laços sociais e afetivos, como uma teia que lhes proporciona acolhimento, sendo facilitado por profissionais no âmbito da atenção básica de saúde s-6 $^{5}$

Deve-se ainda levar em conta que existem componentes que podem agravar a situação enfrentada, tais como a baixa renda econômica, mínimo nível escolar, desestrutura familiar, atuando como barreira na adesão a práticas de promoção da saúde ou de hábitos de vida mais saudáveis. Desta forma, é importante e necessária a articulação da saúde mental com a rede de cuidados, que pode ser composta pelos serviços de saúde, educação e assistência social?

A rede de cuidados pode ser compreendida na dimensão estrutural ou institucional, e na dimensão pessoal, sendo constituída por equipamentos efetivamente importantes para as famílias, que estabelecem vínculos sociais e parcerias por meio dessas ligações. As redes de cuidado podem ser interligadas por serviços de saúde, instituições religiosas, escola, e outros serviços, constituindo uma rede de apoio social que contribui expressivamente com as famílias no enfrentamento das adversidades que surgem no decorrer do cuidado e refletem positivamente no equilíbrio familiar e na qualidade de vida dos membros envolvidos nesse processo ${ }^{7}$.

A Rede de Atenção Psicossocial (RAPS) tem por finalidade a criação, ampliação e articulação de pontos de atenção à saúde para pessoas com sofrimento ou transtorno mental e com necessidades decorrentes do uso de crack, álcool e outras drogas, no âmbito do Sistema Único de Saúde (SUS) e apresenta como um de seus objetivos específicos promover cuidados em saúde especialmente para grupos mais vulneráveis (criança, adolescente, jovens, pessoas em situação de rua e populações indígenas). Embora a atenção em saúde mental seja tarefa de uma rede articulada de serviços, essa articulação deve incorporar os recursos da comunidade para se constituir em verdadeiros espaços de inclusão na cidade destinados às pessoas com transtornos mentais ${ }^{5-6}$.

De acordo com o Ministério da Saúde ${ }^{7}$ a rede de cuidados em saúde mental infantojuvenil deve ser composta por diversas ações e serviços: ações de saúde mental na Atenção Primária, Centros de Atenção Psicossocial (CAPSi), ambulatórios, centros de convivência e cultura, acesso ao lazer e à escola.

A Política de Saúde Mental para a infância e adolescência prevê a assistência especializada para crianças em intenso sofrimento psíquico, nos Centros de Atenção Psicossocial Infantojuvenil (CAPSi), que são serviços de referência da Rede de Atenção Psicossocial, de base comunitária, responsáveis por um determinado território. Acolhem crianças e adolescentes com transtornos mentais e/ou com problemas em decorrência do uso de álcool, crack e outras drogas. O CAPSi deve compartilhar responsabilidades com outros serviços da rede de saúde mental e demais equipamentos de saúde do município ou região, além de desenvolver ações intersetoriais com a educação, a justiça e a assistência social $^{1,3}$.

$\mathrm{Na}$ Atenção Primária, o desenvolvimento de intervenções em saúde mental é construído no cotidiano 
dos encontros entre profissionais e usuários, visando criar estratégias e construir o cuidado em saúde. A Política Nacional de Atenção Básica tem na Estratégia de Saúde da Família (ESF) a porta de entrada do modelo de atenção à saúde, e o Núcleo de Apoio a Saúde da Família (NASF) como equipe apoiadora, para o desenvolvimento de ações compartilhadas, que promovem a ampliação da clínica, auxiliam no aumento da capacidade de análise e de intervenção sobre problemas e necessidades de saúde da comunidade ${ }^{6}$.

$\mathrm{O}$ terapeuta ocupacional tem conquistado e consolidado espaço em programas que atuam diretamente na comunidade. Priorizar o social em reabilitação psicossocial implica sair de centros de reabilitação, de hospitais e de oficinas abrigadas para criar espaços de intervenção na própria comunidade. Portanto, deve intervir sobre os problemas e relações dos indivíduos na comunidade, compreendendo o papel das atividades como importante fator na emancipação e na construção de histórias e contextos ${ }^{5,8}$.

Segundo Ribeiro9, "A Terapia Ocupacional não deve ser apenas um instrumento de intervenção para controle e eliminação do mal estar psíquico, ela deve contribuir para que a vida coletiva e as existências individuais sejam mais interessantes, abertas e criativas".

O terapeuta ocupacional se insere na atenção básica a partir do NASF, e desenvolve ações de prevenção e promoção da saúde. Essas ações facilitam a independência e a participação social das pessoas frente à diversidade de condições e as necessidades presentes no cotidiano.

$\mathrm{O}$ estudo objetivou conhecer a rede de cuidado das crianças e adolescentes em sofrimento psíquico e descrever as ações que visem à promoção, recuperação da saúde mental, bem como a minimização do sofrimento a partir de uma intervenção territorial com atuações específicas da Terapia Ocupacional e da equipe de saúde da família. A motivação para a realização do estudo surgiu a partir do conhecimento das pesquisadoras sobre as condições de saúde, educação e lazer do público em questão, obtidas a partir das vivências no projeto. As pesquisadoras atuavam na área por meio de disciplinas práticas do currículo pedagógico, fazem parte do quadro de docentes do curso de Terapia Ocupacional da Universidade Federal de Pernambuco e conduziram a pesquisa juntamente com discentes do curso. Foram identificadas as condições socioeconômicas e demográficas dessa clientela; as relações interfamiliares e com a rede de cuidados e a participação no brincar, na escola e na comunidade.
As ações da pesquisa e da intervenção fizeram parte do "Projeto de Pesquisa-ação: ações interdisciplinares na atenção a pessoas em situações de vulnerabilidade social". Foram desenvolvidas por uma docente terapeuta ocupacional e graduandos do curso de Terapia Ocupacional na perspectiva da atenção primária à saúde.

\section{MÉTODO}

Trata-se de um estudo de abordagem qualitativa, baseado no desenho da pesquisa-ação. Este tipo de pesquisa se constitui na lógica entre a teoria e a prática e intervém numa situação real produzindo conhecimento útil e relevante ${ }^{11}$. Para o processo de pesquisa-ação acontecer, houve uma pactuação entre o respectivo distrito sanitário e a universidade, a fim de se estabelecer a pesquisa e também as ações de promoção e proteção da saúde, via os projetos de extensão que promovem as ações. A pesquisa-ação ocorreu desde o momento da identificação dos casos, implicando e sensibilizando profissionais para a problemática da saúde mental no território, assim como no momento de coleta das informações, pois os participantes, ao serem perguntados sobre a rede de cuidados, acabaram por refletir sobre sua condição de vida e seus problemas, sendo convocados a buscar soluções, que serviram como base para as ações de cuidado realizadas em corresponsabilização com usuários e profissionais.

O estudo foi realizado com crianças e adolescentes com história de sofrimento psíquico e seus familiares, cadastrados em quatro unidades de saúde do Distrito Sanitário IV, pertencentes à cidade do Recife, no estado de Pernambuco.

Para que os participantes fossem identificados na comunidade, os Agentes Comunitários de Saúde (ACS) foram sensibilizados e capacitados por meio de cartilhas e palestras contendo orientações acerca das características e peculiaridades do sofrimento psíquico na infância e adolescência, bem como das situações de risco e vulnerabilidade para instalação de quadros de sofrimento no futuro. A capacitação aconteceu em dois encontros na Unidade de Saúde da Família e fez parte da proposta do projeto de pesquisa. Nesses momentos foi possível esclarecer as dúvidas que os ACS apresentavam quanto aos possíveis casos detectados em suas microáreas.

A coleta de dados foi realizada nos domicílios e em espaços da comunidade, selecionados para as intervenções territoriais no período de setembro de 2012 a abril de 2013. Para tal, utilizou-se uma entrevista semiestruturada contendo dados socioeconômicos, demográficos, e as relações na rede familiar e de cuidados. 
Os dados sobre aspectos socioeconômicos e demográficos foram colhidos com os familiares responsáveis e em seguida sistematizados para a caracterização e descrição dos participantes. Já as informações das relações na rede familiar e de cuidados foram respondidas pelas crianças e adolescentes, sendo representadas por meio do ecomapa. Posteriormente, os fluxogramas individuais de todos os participantes foram comparados e analisados, sendo construído um ecomapa único, representativo da maioria das relações.

O ecomapa se configura em um instrumento útil para avaliar as relações familiares com o meio social. É representado por diagramas interligados por um círculo ao meio representando a família ou mesmo o indivíduo, pelo fluxo das relações e a probabilidade de relações sociais da família, força da ligação, o impacto sobre a família e a qualidade da ligação. O estudo do ecomapa permite analisar as possibilidades de atuação conforme as atividades desenvolvidas e a rede social que participa do cotidiano da família ${ }^{12}$.

As informações da entrevista e as observações durante as visitas domiciliares subsidiaram as intervenções no território.

Para as intervenções utilizou-se como referencial as diretrizes do Núcleo de Apoio à Saúde da Família (NASF); nesta perspectiva realizaram-se visitas domiciliares para o acolhimento das demandas de cuidado e então foram construídos os ecomapas dos usuários para o direcionamento das ações. As intervenções de orientação às professoras, discussão de casos, adaptação do material escolar nas escolas também fizeram parte das ações, a fim de criar estratégias para melhorar o desempenho escolar da população estudada ${ }^{10}$.

Foi desenvolvido diário de campo para descrição das observações das intervenções realizadas no domicílio e na comunidade para conduzir o planejamento das ações de cuidado. Os achados sobre a rotina e relações das crianças e adolescentes foram categorizados em caracterização dos participantes, rede de cuidados e relações familiares, e as intervenções realizadas, discutidas com base no levantamento da bibliografia científica.

Por se tratar de populações vulneráveis, tanto devido às possibilidades diagnósticas como porque tais participantes não têm autonomia legal para decidirem sua participação na pesquisa, os Termos de Consentimento Livre e Esclarecido (TCLE) foram assinados pelos participantes e responsáveis. A pesquisa foi aprovada do projeto pelo Comitê de Ética em Pesquisa do Centro de Ciências da Saúde da UFPE, $n^{\circ}$ do CAEE 02666512.6.0000.5208, no dia 23 de julho de 2012 .

\section{RESULTADOS E DISCUSSÃO}

Será apresentada, inicialmente, a caracterização dos participantes, de acordo com as condições socioeconômicas e demográficas. Posteriormente apresenta-se, por meio do ecomapa, a rede de cuidados e as relações familiares e, em seguida, descreve-se o processo de intervenção no território.

\section{Caracterização da população}

Fizeram parte do estudo crianças e adolescentes encaminhadas pelos Agentes Comunitários de Saúde (ACS) por apresentarem situação de risco e vulnerabilidade social, problemas comportamentais, dificuldade na aprendizagem, relação conflituosa com a família e na comunidade. O grupo estudado foi composto por quinze indivíduos com faixa etária entre 6 e 17 anos; destes, dez são do sexo masculino e cinco do sexo feminino, sendo nove adolescentes.

Segundo Poeta e Rosa Neto ${ }^{13}$, a adolescência predispõe ao surgimento de sintomas e da instalação de quadros de transtorno mental. Sabe-se que é uma fase marcada por constantes transformações psíquicas e de conflitos internos, inclusive no âmbito escolar. Todavia a intensidade e o histórico de sofrimento, o contexto no qual ocorre, a atmosfera de vida desses jovens, bem como suas características individuais e dos cuidadores, são fatores que conformam o prejuízo sobre sua saúde mental ${ }^{14}$.

Quatorze dos participantes estavam inseridos no contexto escolar, sobretudo em escolas e classes regulares. Os participantes mantinham suas atividades escolares em classes regulares e assim experimentavam dificuldades e conflitos decorrentes do sofrimento psíquico e do estigma, mas também vivenciavam a oportunidade de estar inseridos socialmente e de conviver com seus pares, num ambiente onde a oferta de estímulos para um desenvolvimento sadio é frequente.

O nível socioeconômico das famílias era predominantemente baixo, sendo a renda salarial familiar de um salário mínimo e sem haver vínculo empregatício em treze dos casos estudados. Tal situação tem sido apontada na literatura como fator de risco para a saúde mental. Nascer na pobreza, bem como viver em ambiente psicologicamente desfavorável, são condições de alto risco para a saúde mental das pessoas, e alguns indivíduos não conseguem desenvolver habilidades para um bom desenvolvimento psicoemocional ${ }^{15}$.

Embora os genitores tenham aparecido, em oito dos casos, como os principais responsáveis pelas crianças 
e adolescentes, foi possível constatar que os avós, especialmente os maternos, eram os principais apoiadores e cuidadores. Nos casos em que a responsabilidade pela criança ou adolescente foi conferida a outros, os avós foram vistos como principal fonte de apoio aos pais que precisavam trabalhar para garantir a renda salarial familiar ou mesmo porque se sentiam corresponsáveis no cuidado dos netos ${ }^{16}$.

\section{Rede de cuidado e relações familiares}

A análise do ecomapa (Figura 1) mostra a fragilidade da relação dos usuários com os serviços de atenção especializada e a forte relação com as Unidades de Saúde da Família (USF); isto aponta a efetividade das ações da Atenção Básica, conforme preconizado no SUS, ao introduzir o Programa de Saúde da Família na perspectiva de humanizar as práticas de saúde por meio de um vínculo entre os profissionais de saúde e a população. ${ }^{17}$ A Estratégia de Saúde da Família tem sido reconhecida como uma política de sucesso por permitir a proximidade do usuário com os profissionais das unidades de saúde, visto que estes possuem essencialmente o conhecimento das necessidades locorregionais bem como dos equipamentos da rede capazes de fornecer suporte a essas demandas, o que favorece a resolutividade das dificuldades encontradas no território ${ }^{18,19}$.

Atividades como o brincar e o lazer estavam sendo pouco vivenciadas pelas crianças e adolescentes. A restrição do espaço físico e a violência foram apontadas pelos responsáveis como fatores dificultadores dessas atividades.
Para Motta e Enumo ${ }^{20}$, ao brincar, a criança altera o ambiente em que se encontra, aproximando-o de uma realidade idealizada, o que pode ter um efeito bastante positivo em relação ao enfrentamento de sua realidade. Com isso, a própria atividade recreativa, livre e desinteressada, tem um efeito positivo, quando se considera favorável tudo aquilo que auxilia na promoção do bem-estar da criança. Durante o brincar, a ação tornase mais reveladora do que a própria palavra não dita, uma vez que este fazer é carregado de conteúdo simbólico em que as crianças retomam concepções. Além disso, as atividades lúdicas criam possibilidades para que o inconsciente se expresse por meio dos atos psicomotores e sensoriais. As limitações encontradas pelos participantes o restringiam e muitas vezes os impossibilitavam de desenvolverem habilidades inerentes a suas fases de desenvolvimento.

As relações com a vizinhança e amigos são apontadas pelos participantes como sólidas e de apoio permanente à população do estudo. Quando a família não possui uma rede de suporte, as dificuldades se somam às do cotidiano, visto que as pessoas, quando estão passando por um período de sofrimento, esperam contar com suporte, sejam os vizinhos, comunidade, igreja, entre outros. Desta forma, torna-se fundamental ampliar a intervenção para a vizinhança, por compreender esta como um recurso importante da rede de cuidados à criança e ao adolescente em sofrimento psíquico, conforme estabelece o Ministério da Saúde ao dispor sobre as diretrizes de ações na Atenção Primária de Saúde. As ações de cuidado devem extrapolar o núcleo familiar no sentido de manter e fortalecer o vínculo da família com a comunidade ${ }^{10,21}$.

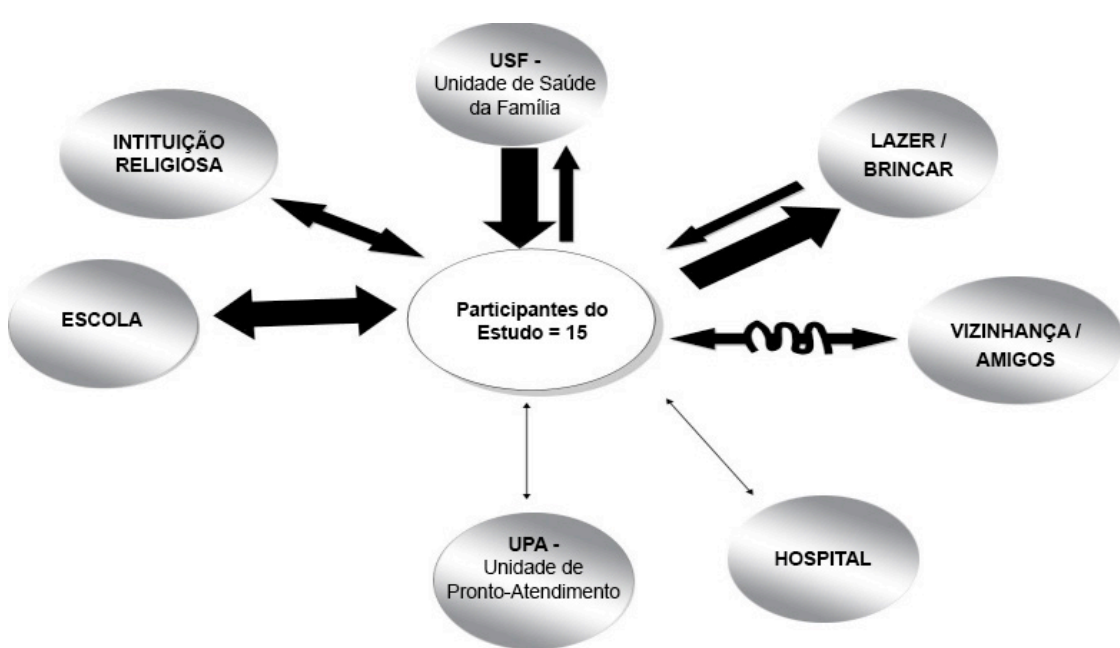

Figura 1 - Ecomapa da rede de cuidado dos participantes

\section{LEGENDA:}

Fluxo e permuta de forças

Equilibrado: as setas incidem igualmente para ambos os lados

Desequilibrado: seta incidindo mais fortemente para um dos lados

Não há troca: setas incidem só para um lado

Intensidade das relações

Forte:

Média:

Superficial:

Interrompida: - - - - - - -

Conflituosa: $A A A$ 
No tocante ao estudo das relações familiares (Figura 2), identificou-se a família como mantenedora de vínculos fortes entre estas crianças e adolescentes. Em contrapartida, havia diversos conflitos intrafamiliares e muitos dos sintomas que os participantes apresentavam podem estar relacionados a uma dinâmica familiar conturbada, inclusive com episódios de violência.

Nesse contexto, o ambiente doméstico torna-se um local potencialmente empobrecido para o desenvolvimento saudável na infância e adolescência, visto que a família serve como modelo para o aprendizado de padrões comportamentais e sociais dos filhos. Dessa forma, quando existem episódios de violência, a família atua como um modelo negativo, gerando comportamentos prejudiciais à interação social e à saúde mental das crianças e adolescentes que convivem com essas práticas ${ }^{16}$.

Também foi observado que muitos usuários mantinham com o pai uma relação superficial, que pode estar relacionada à baixa quantidade e qualidade no cuidado, explicada por alguns como "falta de tempo pelo excesso de trabalho", e, quando a relação existia, era predominantemente conflituosa. A carência da figura paterna no cotidiano familiar gera uma sobrecarga materna e acúmulo de tarefas, dificultando os cuidados com a educação e o suporte emocional, podendo aumentar o risco de problemas escolares, comportamentais e emocionais ${ }^{22}$.

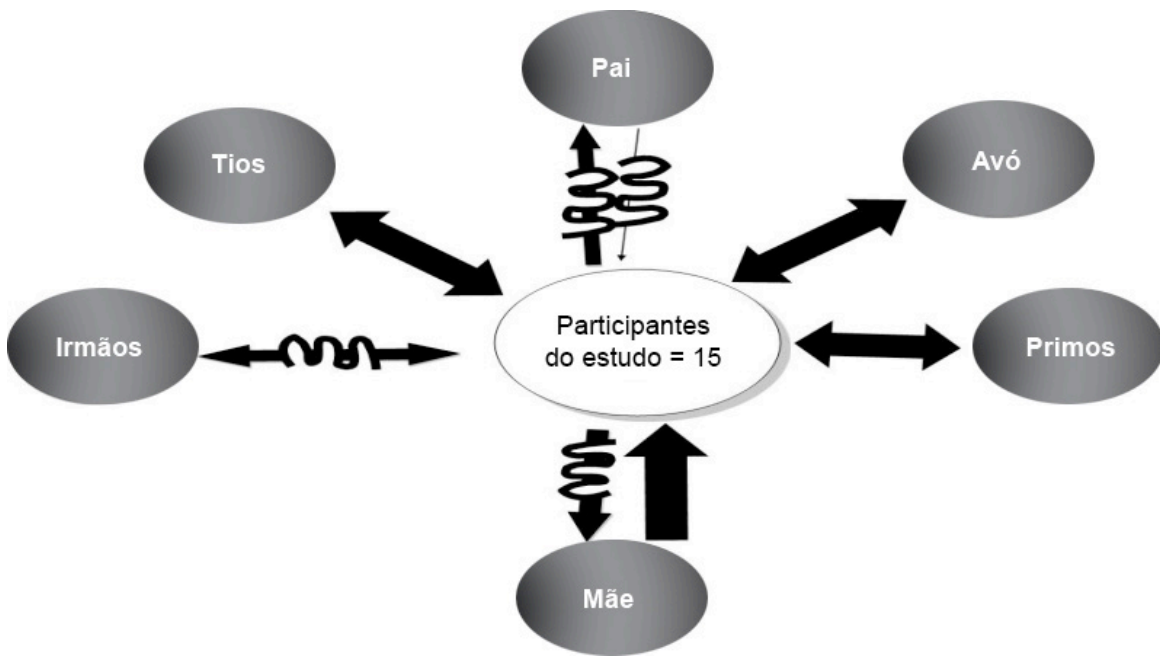

Figura 2 - Ecomapa das relações familiares

\section{LEGENDA:}

Fluxo e permuta de forças

Equilibrado: as setas incidem igualmente para ambos os lados

Desequilibrado: seta incidindo mais fortemente para um dos lados

Não há troca: setas incidem só para um lado

Intensidade das relações

Forte:

Média:

Superficial:

Interrompida: - - - - - - -

Conflituosa:

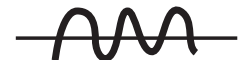

\section{Intervenções no território}

Participaram das intervenções a terapeuta ocupacional responsável pela pesquisa, e também alunos do curso de Terapia Ocupacional da UFPE, integrantes do grupo de pesquisa. As intervenções tiveram como foco a inclusão da criança ou adolescente no território visando à participação social e a autonomia. Para isso, foi necessário fazer o mapeamento dos serviços de apoio contidos na rede de cuidados e se articular com eles. Para melhor assistir à criança e à família, foram realizadas ações matriciais com os CAPSi e USF da área para discussão de caso e possíveis encaminhamentos (Quadro 1). Ao perceber as dificuldades de acesso ao lazer e por entender os prejuízos que isto acarreta ao desenvolvimento, foi possível criar, em parceria com a USF local, um projeto de brinquedoteca comunitária, e a comunidade se organizou para implantála. De acordo com o Ministério da Saúde ${ }^{10}$, é atribuição da Equipe de Saúde da Família incentivar a criação de espaços de inclusão social, ricos em estímulos, com ações que ampliem o sentimento de pertinência social, por meio das ações referentes às práticas de educação e lazer.

Ao identificar que as relações interpessoais eram conflituosas, um dos focos prioritários da intervenção no domicílio foi o fortalecimento do vínculo familiar (Quadro 1). A relação desestruturada das famílias causava, muitas vezes, uma atmosfera cercada de sentimentos não compreendidos e negativos entre os membros, que reforçavam as dificuldades e repercutiam na autoestima dos participantes. 
Quadro 1 - Descrição das intervenções realizadas no território. Recife, PE, Brasil 2013

\begin{tabular}{|l|}
\hline \multicolumn{1}{|c|}{ Intervenções na rede de cuidados } \\
\hline Mapeamento dos dispositivos de apoio e articulação com tais serviços; \\
\hline Encaminhamento para os serviços especializados; \\
\hline Ações matriciais com os CAPSi e USF; \\
\hline Criação de um projeto de brinquedoteca comunitária; \\
\hline \multicolumn{1}{|c|}{ Intervenções no domicílio } \\
\hline Fortalecimento do vínculo familiar; \\
\hline Criação de acordos de convivência; \\
\hline Incentivo à organização da rotina; \\
\hline Realização de atividades autoexpressivas; \\
\hline Confecção de materiais educativos para estimulação dos filhos; \\
\hline Verificação do desempenho escolar; \\
\hline $\begin{array}{l}\text { Adaptações do material escolar, orientações para um melhor } \\
\text { aproveitamento pedagógico. }\end{array}$ \\
\hline \multicolumn{1}{|c|}{ Intervenções no contexto escolar } \\
\hline $\begin{array}{l}\text { Realizadas orientações sobre como identificar e lidar com crianças e } \\
\text { adolescentes em sofrimento psíquico; }\end{array}$ \\
\hline Oferecidas estratégias de atividades que facilitam o aprendizado; \\
\hline Discussão de casos e planejamento de ações; \\
\hline Realizadas oficinas sobre a sensopercepção e experiências sensoriais. \\
\hline
\end{tabular}

Segundo Vieira e Sá ${ }^{21,23}$, os efeitos da intervenção terapêutica ocupacional têm melhores resultados quando os familiares são trazidos para o processo terapêutico com um papel central. A partir disto, as famílias foram estimuladas a criar acordos de convivência, quanto à organização da rotina, e a realização de atividades autoexpressivas; além disso, foram confeccionados materiais educativos contendo orientações aos responsáveis sobre como estimular seus filhos e sobre a importância de se estabelecer uma relação afetiva com os mesmos.

Durante as entrevistas, os familiares relatavam queixas quanto ao desempenho escolar e social dos participantes. Com isso, durante as visitas, a terapeuta ocupacional solicitava o material escolar da criança/ adolescente para se verificar o andamento das atividades educativas. Para aqueles que necessitavam de recurso auxiliar por apresentarem baixa visão ou hiperatividade que lhes dificultava a boa percepção do material, foram então feitas adaptações, além de orientações para um melhor aproveitamento pedagógico, e para o estabelecimento de um espaço e horários para o estudo no domicílio, criando-se uma rotina e estimulando-se a maior participação dos responsáveis. Percebeu-se que muitos dos responsáveis das crianças e adolescentes do estudo possuíam baixa escolaridade e isso foi um fator dificultador para o investimento nas atividades escolares dos filhos ${ }^{16}$.

A escola, embora não seja o único espaço, é um lugar instituído para promover o aprendizado formal. Sua função não se restringe apenas aos processos sistemáticos de ensino-aprendizagem, mas se constitui em importante espaço de trocas, de transmissão de valores sociais e culturais. O papel ocupacional do estudante é geralmente definido pela escola e pelas leis que regulamentam o funcionamento escolar, portanto esta instituição se torna um sistema social em que as crianças aprendem regras de convivência e habilidades acadêmicas ${ }^{23}$.

Nesta perspectiva, viu-se a necessidade de as ações se estenderem também para o ambiente escolar, a fim de proporcionar às crianças e adolescentes cuidado integral em todas as áreas de seu desempenho ocupacional (Quadro 1). Salienta-se então a importância de desenvolver um trabalho com os educadores, os alunos, os pais, a comunidade, que deve se estender para além do espaço da escola, cuja finalidade é colaborar com o processo de aprendizagem, socialização e para o exercício da cidadania ${ }^{23}$.

Dessa forma, ações intersetoriais são necessárias, integrando-se ações da saúde e da educação. No ambiente escolar a Terapia Ocupacional focaliza a melhoria da capacidade de a criança registrar, processar e integrar as 
informações por meio da intervenção direta, da adoção de estratégias compensatórias para a criança e da adaptação do ambiente, a fim de minimizar suas dificuldades e realçar suas potencialidades ${ }^{10,23}$.

Durante as intervenções, percebeu-se discrepância entre as habilidades perceptocognitivas das crianças/ adolescentes e o que lhes era ofertado no âmbito escolar. Alguns dos professores relataram não estar aptos a ensinar a tal público, o que gera nesses formadores angústia e sentimentos de incapacidade. A partir do observado, as intervenções realizadas nas escolas foram de orientações aos professores sobre como identificar e lidar com alunos em sofrimento psíquico e, consequentemente, com dificuldades de compreender as atividades pedagógicas, além da indicação de diferentes possibilidades e estratégias de atividades que facilitam o aprendizado e o interesse pelo conteúdo. Foram realizadas também oficinas com os professores sobre a sensopercepção e experiências sensoriais, para que compreendessem os ganhos que a estimulação sensorial poderia repercutir sobre a aprendizagem e melhorar a participação dos alunos na sala de aula.

\section{CONCLUSÃO}

Durante o estudo se verificou a aplicabilidade e efetividade de algumas ferramentas que podem ser utilizadas pelos profissionais inseridos na atenção primária, como: a realização de práticas de promoção

\section{REFERÊNCIAS}

1. Couto MCV, Duarte ECS, Delgado PGG. A saúde mental infantil na saúde pública brasileira: situação atual e desafios. Rev Bras Psiq. 2008;1(30):390-8. Disponível em: http:// www.scielo.br/pdf/rbp/v30n4/a15v30n4.pdf.

2. Tanaka OY, Ribeiro EL. Ações de saúde mental na atenção básica: caminho para ampliação da integralidade da atenção. Cien Saúde Coletiva (Rio de Janeiro). 2009;14(2):477-86. DOI: http://dx.doi.org/10.1590/S141381232009000200016 .

3. Florey L. Disfunção psicossocial na infância e adolescência. In: Neistadt ME, Crepeau EB. Willard \& Spackman: terapia ocupacional. 11a ed. Rio de Janeiro: Guanabara Koogan; 2002. p.580-91. da saúde e o desenvolvimento de ações clínicas, além da articulação e mapeamento de dispositivos na rede e de estratégias coletivas realizadas nas escolas, nos domicílios e em outros espaços da comunidade que servem como equipamentos de apoio e de promoção da saúde.

Foi possível constatar também as necessidades na efetivação do brincar, as dificuldades escolares presentes na maioria dos casos e as relações familiares conflituosas que prejudicam o desenvolvimento saudável dessa população. Foi observado que a maioria dos participantes vive com poucos direitos de cidadania garantidos, uma vez que lhes são impossibilitados o acesso ao saneamento adequado, à moradia regular, ao lazer, aos serviços especializados de saúde e aos meios de transporte eficientes.

A realidade das configurações e relações familiares conflituosas tem ocasionado a falta de referência para crianças e adolescentes, que estão iniciando suas vidas e começando a descobrir o mundo e sua identidade. Assim, acabam expressando sentimentos negativos por meio de atitudes e comportamentos; inclusive, chegam ao adoecimento. Sabe-se que a família é a primeira e principal instituição social responsável pela formação da personalidade da criança. Uma falha nessa relação acarreta agravos à condição psíquica das crianças. Dessa forma, faz-se fundamental a extensão das ações para os familiares, objetivando-se melhorar os vínculos intrafamiliares e na comunidade, para que todos se apropriem como sujeitos corresponsáveis em seu processo de cuidado.
4. Lambertucci MCF, Magalhães LC. Terapia ocupacional e transtornos invasivos do desenvolvimento. In: Camargos JW. Transtornos invasivos do desenvolvimento: $3^{\circ}$ milênio. Brasília: COORDE; 2002. p.227-35.

5. Brasil. Ministério da Saúde. Portaria No 3.088, de 23 de dezembro de 2011. Institui a Rede de Atenção Psicossocial para pessoas com sofrimento ou transtorno mental e com necessidades decorrentes do uso de crack, álcool e outras drogas, no âmbito do Sistema Único de Saúde. Brasília; 2011. Disponível em: http://bvsms.saude.gov.br/bvs/saudelegis/ gm/2011/prt3088_23_12_2011_rep.html.

6. Vicentin MCG. Infância e adolescência: uma clínica necessariamente ampliada. Rev Ter Ocup Univ São 
Paulo. 2006;17(1):10-7. DOI: http://dx.doi.org/10.11606/ issn.2238-6149.v17i1p10-17.

7. Mendes EV. As redes de atenção à saúde. Brasília: Organização Pan-Americana da Saúde; 2011. p.549-54. Disponível em: http://www.conass.org.br/pdf/Redes_de_ Atencao.pdf.

8. Benetton MJ. Terapia ocupacional e reabilitação psicossocial: uma relação possível? Rev Ter Ocup Univ São Paulo. 1996;4(7):53-8. Disponível em: http://www.jobenetton.pro. br/rebili.htm.

9. Ribeiro MC, Machado AF. A terapia ocupacional e as novas formas do cuidar em saúde mental. Rev Ter Ocup Univ São Paulo. 2008;19(2):72-5. DOI: http://dx.doi.org/10.11606/ issn.2238-6149.v19i2p72-75

10. Brasil. Ministério da Saúde. Secretaria de Atenção à Saúde. Departamento de Atenção Básica. Diretrizes do NASF: Núcleo de Apoio a Saúde da Família. Brasília: Ministério da Saúde; 2010. (Cadernos de Atenção Básica, n. 27, série B. Textos Básicos de Saúde). Disponível em: http://bvsms. saude.gov.br/bvs/publicacoes/diretrizes_do_nasf_nucleo. pdf.

11. Thiollent M. Metodologia da pesquisa-ação. São Paulo: Cortez; 2009.

12. Brasil. Ministério da Saúde. Saúde mental - matriciamento. Brasília: Ministério da Saúde, Centro de Estudo e Pesquisa em Saúde Coletiva; 2011. p.219-35.

13. Poeta LS, Rosa FN. Estudo epidemiológico dos sintomas do transtorno do déficit de Atenção/hiperatividade e transtornos de comportamento em escolares da rede pública de Florianópolis usando a EDAH. Rev Bras Psiquiatr. 2004;26(3):150-6. DOI: http://dx.doi.org/10.1590/S151644462004000300004

14. Assis SM, Avanci JQ, Pesce RP, Ximenes LF. Situação de crianças e adolescentes brasileiros em relação à saúde mental e à violência. Ciên Saúde Coletiva. 2009;14(2):319-61. http://dx.doi.org/10.1590/S1413-81232009000200002
15. Ludermir AB. Desigualdades de classe e gênero e saúde mental nas cidades. Physis - Rev Saúde Coletiva. 2008;18(3):451-67. DOI: http://dx.doi.org/10.1590/S010373312008000300005 .

16. Halpern R, Figueiras ACM. Influências ambientais na saúde mental da criança. J Pediatr (Rio J.). 2004;80(2):104-10. DOI: http://dx.doi.org/10.1590/S0021-75572004000300013.

17. Brasil. Ministério da Saúde. Secretaria de Assistência à Saúde. Coordenação de Saúde da Comunidade. Saúde da família: uma estratégia para a reorientação do modelo assistencial. Brasília: Ministério da Saúde; 1997. Disponível em: http://bvsms.saude.gov.br/bvs/publicacoes/cd09_16.pdf.

18. Organização Pan-Americana da Saúde. A atenção à saúde coordenada pela APS: construindo as redes de atenção no SUS: contribuições para o debate. Brasília: Organização PanAmericana da Saúde; 2011. Disponível em: http://apsredes. org/site2012/wp-content/uploads/2012/03/aps_verde_new. pdf.

19. Daguano LQ, Fantacini RAF. O lúdico no universo autista. Linguagem Acad (Batatais). 2011;1(2):109-22. Disponível em: file:///C:/Users/Administrador/Downloads/sumario7. pdf.

20. Mota AB, Eunumo SRF. Brincar no hospital: estratégia de enfrentamento da hospitalização infantil. Psicol Estud (Maringá). 2004;9(1):19-28. Disponível em: http://www. scielo.br/pdf/pe/v9n1/v9n1a04.

21. Viera CS, Mello DF, Oliveira BRG, Furtado MCC. Rede e apoio social familiar no seguimento do recém-nascido prétermo e baixo peso ao nascer. Rev Eletr Enf. 2010;12(1): 11-9. http://www.fen.ufg.br/revista/v12/n1/v12n1a02.htm.

22. Taylor RD, Wang MC. Resilience across contexts: family, work, culture and community. New Jersey: Lawrence Erlbaum Associates; 2000.

23. Sá DGF, Bordim IAS, Martin D, Paula CS. Fatores de risco para problemas de saúde mental na infância/adolescência. Psicol Teor Pesq. 2010;26(4):643-52. Disponível em: http:// www.scielo.br/pdf/ptp/v26n4/08.pdf. 